\title{
BACKGROUND
}

Intense global migration in some parts of the world, present particular challenges in oncology healthcare, due to the social and cultural demands this places on host services (Weber et al., 2016). Culture is an important social determinant of health, which can influence access and delivery of safe and appropriate health services (Surbone, 2008).

The individual's cancer journey can be one of the most distressing human experiences, evoking complex emotional responses including denial, anxiety, and fear which may affect a survivors' psychological well-being (Cordova et al., 2017; Lu et al., 2017). Cultural differences/ barriers are added complexity which is strongly associated with quality of life, acceptance of life-prolonging measures, reluctance to withdraw life support, and impact on the quality of communication (Schrank et al., 2017; Tartaglione et al., 2018). Typically, gaining awareness of culture commences with awareness of one's own cultural beliefs and identifying potential biases and prejudice through self-reflection and self-critique (Rao et al., 2018). This combined with knowledge of different cultural attitudes, lifestyles and practices should enable health care providers to take account of individual preferences, positively impacting communication and decision-making (Markova and Broome, 2007). However, the complex dynamics arising from racial and ethnic differences, the educational status and language barriers create a challenging environment in which health care providers are required to operate (Rao et al., 2018). This has inevitable implications for oncology care and decision-making processes, all of which can be affected by beliefs and attitudes about health and disease, traditions associated with acceptance of life-prolonging measures and withdrawal of life support (Vallurupalli et al., 2012). This, in turn, can have a major impact on diagnosis, appropriate treatment, survivor and family satisfaction, and quality of care (Palmer et al., 2014). 
In oncology settings, cultural competency requires specific knowledge and clinical skill that support nursing activities (Huang et al., 2008; Sulstarova, 2016). Culturally competent nursing care is seen to contribute to the reduction of health disparities through assessment of survivor's health beliefs and cultural needs using the complex combination of cultural knowledge, awareness, attitudes, and skills (Douglas et al., 2014). The beliefs and perspectives of nurses are characterized by a willingness to provide effective care for people from different cultures (Brown et al., 2016; van Eechoud et al., 2016). Culturally competent nursing care can help overcome the risks of cultural humiliation, racism, classism, sexism, ageism, and stereotyping in clinical practice by providing empathetic, respectful, and effective health care (Rao et al., 2018). Cultural competence is a dynamic, lifelong learning process (Moore et al., 2010), requiring the combining of specific knowledge, clinical skills, and attitudes that enhance the therapeutic effects throughout the cancer care journey (Surbone, 2008). According to Sharifi and colleagues (2019) cultural competence involves cultural awareness, cultural knowledge, cultural sensitivity, cultural skill, cultural proficiency, and dynamicity (Sharifi et al., 2019). To be able to care in culturally competent ways there is also a moral obligation to protect human rights as health disparities can arise as a result of different value systems and power dynamics which may cause negative motivation, emotions, quality of life and care (You et al., 2018).

Within the provision of oncologic healthcare services, nurses as 24-h care providers typically have the closest contact and play a key role in meeting the needs of survivors through effective communication to achieve culturally qualified care and the provision of support (Sheldon et al., 2012). In the field of mental health, cultural competency in nursing includes the specific skill of adapting treatments and practices to better match the particular needs of service users and considers issues such as values, assumptions, practices, communication styles, group norms, biases, and personal experiences (Nardi et al., 2012). It is 
important for nurses to examine cultural expressions of diverse populations while providing culturally sensitive health care that will optimize health outcomes (Rosalie and Sandra, 2013). Nurses previous experience of culture has the potential to impact their approach to cultural care (Huang et al., 2009). Understanding the nurses and cancer survivors' perspective on which factors influence culturally competent care is important in the development of diverse healthcare services. This qualitative study aimed to understand how both survivors and nurses define cultural care needs in cancer care and make recommendations toward improvements in nursing practice.

In relation to the terminology, we have employed the word 'survivor' rather than 'patient' to represent those who participated in this study. This is consistent with the National Coalition for Cancer Survivorship (NCCS) definition of survivor as "any person diagnosed with cancer, from the time of initial diagnosis until his or her death. This expansive definition of "survivor" includes people who are dying from untreatable cancer" (NCCS, 2019). This also addresses preferences not to be defined by a medical label. However, the term patient appears in the questionnaire and indirect quotes, in order to remain true to the data.

\section{Theoretical Framework for the study}

The study adopted Leininger's Transcultural Nursing Theory, which considers the diverse and common dimensions of cultural caring. This theory posits that care is the central, dominant and distinctive feature in cultural nursing and helps nurses develop cultural sensitivity including anthropological insight, life experiences, beliefs and values (Leininger, 2002). The theory reflecting these components, guides nurses in providing care that is meaningful and fits with cultural beliefs and lifeways of service users. Nurses are encouraged to reflect on their decisions and actions to arrive at culturally congruent care for health, well-being or dying (Leininger, 1991). The first step is to understand that culture and experience has an impact on attitudes, beliefs, values, behaviors. As such it helps nurses identify and improve the skills, 
knowledge, vision, and beliefs relating to cross-cultural nursing care (Leininger, 1991; Leininger, 1999). This theory provided us a better understanding of the cross-cultural issues in cancer experience, which was helpful in informing the structure, content, and focus of the study. It also helped structure both the semi-structured interview questions and interpret the findings of the study.

\section{METHOD}

\section{Study Design}

A qualitative descriptive design was chosen in order to gain insight into cancer survivors' and nurses' perspectives of culturally competent care (Maxwell, 2013). Qualitative descriptive design was considered the most appropriate for an in-depth examination of the participants' experiences (Sandelowski, 2010; Neergaard et al., 2009). The study followed the COREQ (Consolidated criteria for Reporting Qualitative Research) Checklist (COREQ, 2019).

\section{Setting}

This qualitative study was carried out from June to September 2018 in the Oncology Clinics, in one hospital, serving almost 80,000 individuals, in a year. Whilst not specifically targeted for cultural diversity, it was anticipated that there would be sufficient cultural diversity within a hospital population of 80,000 people.

\section{Participants}

A purposive sampling method was used to recruit adults diagnosed with different types and stages of cancer. Survivors were eligible to participate if they were aged 18 years or older, undergoing curative treatment, having bed-rest in hospital, the ability to provide written informed consent and being able to understand and speak Turkish. The eligibility criteria for nurse participants were as follows: having a minimum one-year experience of working in cancer care, being Turkish speaking and not having any serious cognitive impairment that may impede their ability to provide informed consent. The purposive sampling method for the 
identification and selection of participants aimed to provide information-rich data to deeply investigate the issue in accordance with the study's aim. The maximum variation sampling method was the purposive sampling method adopted in this study.

The nurses were recruited from the inpatient wards, providing acute oncological care to adult survivors with any type of cancer. Survivors were treated with curative intention and were receiving chemotherapy and/or radiotherapy. In total, the wards had a bed capacity of 160 distributed on both single and shared rooms. Nurses on the wards worked both day- and evening shifts up to 12-h in length. They cared for 5-8 survivors each.

\section{Data Collection}

The sample size of both groups were determined by achieving theoretical saturation, which is considered to be the point that indicates adequate data has been collected for a detailed analysis (Flick, 2018). To achieve theoretical saturation, the researchers continued sampling and analyzing data until no new data appeared and all concepts were well-developed. Interviews were ended once data saturation had been achieved and no new data or codes were emerging (Flick, 2018).

Eligible survivors and nurses who met the inclusion criteria were identified by the researchers. The participants received written information letter about the study in printed form on the wards. The authors (YSÜÖ and MK) approached the participants to answer their questions regarding the study, and to ask for consent to participate. When consent was obtained, a time and place for the interview were decided. The socio-demographic data presented in tables 1 and 2 were obtained before the interviews were conducted using a questionnaire, based on typical characteristics drawn from an appreciation of previous literature (Lu et al. 2017; Nardi et al, 2012; Rao et al., 2018; Schrank et al., 2017; Tee, 2016; Weber et al., 2016). The characteristics of the participants, included age, gender, educational background and marital status. 
The interviews were conducted in a quiet room at the hospital, by experienced interviewers (YSÜÖ and MK). No other persons were present during the interviews. The interviewer (YSÜÖ) was a female with a Ph.D. degree in psychiatric nursing, also an assistant professor in psychiatric nursing department at a University and had experience in conducting qualitative research. The interviewer (MK) was a female with a Ph.D. degree in psychiatric nursing, also an assistant professor in psychiatric nursing department at a University and also had experience of conducting qualitative research. The interviewer (YSÜÖ) conducted the interviews and asked the semi-structured questions with responses being audio recorded. The interviewer (MK), who had gained informed consent prior to each interview, also took notes of the interviewee's responses, to avoid any audio recording problems such as low battery or voice volume problems. The interviewers were aware of possible unequal power dynamics between the interviewer and the interviewee during the conversation and focused on respectful and empathic behavior (Malterud et al., 2015). There were no prior relationships between the interviewers and the interviewees. The audio-taped interviews lasted $45 \mathrm{~min}$ on average. The transcripts were not returned to the participants for comment as we had both audio recordings and notes to check accuracy.

A semi-structured, pilot-tested, interview guide was used (Fig 1) which was based on themes drawn from previous published literature and adapted according to known issues arising during the cancer process. All interviews were performed in Turkish. In order to avoid unintended interpretation translation from Turkish to English took place after the interviews were completed. The translation was made by an expert academic in the foreign language department of the university, and the edits of translation were made by the third researcher (ST) who is a native British. In this way, this translation was double-checked. Additional information regarding the participants' gender, age, etc. were obtained before commencing the interview. 


\section{Analysis}

All interviews were transcribed using the framework analysis approach that involves a number of highly interconnected and distinct phases (Malterud et al., 2015). These phases include familiarization, identifying a thematic framework, indexing, charting, mapping, and interpretation. Two researchers (YSÜÖ and MK) independently listened and transcribed the interviews and initially read and re-read to gain key ideas and recurring themes (familiarization). Key ideas and recurring themes were analyzed and were labeled into categories (indexing). A summary of categories was produced under each thematic heading (charting). Charts were assembled to show the associations between themes and categories according to the nature of the phenomenon (mapping and interpretation). Interviews were conducted until data saturation had been achieved and no new data or codes emerged. To ensure rigor and to avoid discrepancies, a third researcher (ST) looked over the emerged themes and sub-themes and where necessary referred back to the transcripts to make a final judgment on themes and subthemes. The aim of the study guided the thematization. Where necessary, sub-themes were formed to reflect the complexity of certain themes. Codes and themes were compared and discussed until consensus was reached by the authors (YSÜÖ, ST, and MK), which was a form of analytical triangulation (Fusch and Ness, 2015). In order to avoid unintended interpretation translation from Turkish to English took place after the interviews were completed. The translation was made by an expert academic in the foreign language department of the university, and the edits of translation was made by one of the authors (ST) who is a native British.

\section{Ethical Considerations}

The study adhered to the principles of the Declaration of Helsinki. The study procedures were approved by Hacettepe University Non-Interventional Clinical Research Ethics Board (protocol no. GO 18/514) before it was initiated and the necessary permissions from the 
hospital were obtained. All the participants were informed of the voluntary nature of participation. Verbal and written informed consent was obtained from each participant. Participants could withdraw from the study at any time without stating a reason. All written material and audio copies were stored in a locked safe.

\section{RESULTS}

\section{Characteristics}

Overall, interviews were conducted with twenty-nine survivors and twenty-three nurses.

Nurses were aged $32.26 \pm 5.59$ years; $91.3 \%$ were female; $56.5 \%$ were married; $69.6 \%$ were graduated to BSc level, 52.2\% were working in oncology settings for more than 6 years.

Survivors were aged $52.86 \pm 15.57$ years; $58.6 \%$ were male; $82.8 \%$ were married; $31.0 \%$ were graduated to the MSc level and 31.0\% were graduated to high school level. Of the survivors 27.6\% were diagnosed with colon cancer, and $82.8 \%$ had not experienced a recurrence.

\section{Main Findings}

Three main themes and eight sub-themes were identified. The main themes were: 1) improving culturally competent care, 2) cross-cultural care expectations, and 3) reality in cross-cultural care activities (see Fig 2). The identity of individual participants was anonymized but for clarity, each quote is identified using the signifier ' $S$ ' for the survivor and 'N' for Nurse.

\section{Improving culturally competent care}

Participant feedback shows there were deficits in survivor experiences of care that seemed to stem from lack of awareness, knowledge, and training amongst nursing staff. To be able to prevent these deficits and improve cross-cultural care, participants emphasized the necessity for effective communication, knowledge enhancement related to culture, increasing cultural awareness and not feeling ignored.

\section{a) The Importance of effective communication}


Culturally appropriate connections between survivors and the nurse were linked to positive health outcomes and experiences via increasing effective human to human communication. Survivors from all cultural backgrounds represented in the sample, cited the benefits of improved communication:

"Accepting and respecting the culture of the patient lies at the center of good nurse-patient communication." (S27, 38 years old, F).

Another agreed:

"We all different from each other, even if we share the same diagnosis.... so, communication is essential to meet a person's private and cultural needs. Particularly those people with cancer who may be struggling with treatment or facing the end of their life." (S29, 60 years old, $M)$.

Additionally, some nurses offered insights into how direct survivor-staff communication and providing assistance for survivors to express their cultural expectations improves their wellbeing:

"The first thing I attend to in my care is communication. Communicating with the patient is key... Because we're the ones providing 24-h bedside care and noticing the cultural signs...., we're the ones who understand and appreciate it... Also, it's important that patients do not feel afraid to ask questions or talk with us... This is an essential element in routine care." (N13, 33 years old, $M$ ).

Another recognized that both verbal and non-verbal signs were important:

"As humans, we can understand each other with the help of communication. Our patients are human too, and we only can touch their life by communicating. Understanding the culture is so very important so when a patient suddenly stops communicating verbally, you need to determine what's the reason ...so that you can respond to the cues” (N14, 39 years old, F).

\section{b) Knowledge enhancement related to different cultures}


The purpose of nurses learning about culture is about acceptance of cultural differences through challenging preconceived notions and understanding and tolerating differences. It was suggested by one nurse that this must be addressed during training:

"First-and-foremost, nurses must be educated in cross-cultural care." (S14, 49 years old, F).

The purpose of this is to equip faculty to support effective cultural encounters in the clinical setting. The integration of theory and practice was seen as essential to build confidence and competence:

"Maybe the well-structured and practice-based education can help us to detect the crosscultural needs of patients with cancer. This can also help to build our confidence and be more efficient when caring for patients." (N18, 28 years old, $M)$.

Another suggested there was a need for evidence to inform practice:

"It is important for the nurse to have basic knowledge of the impact of cultural differences and its process... because we're the main caregiver of the patient... So, we can anticipate and identify what is best to meet the patient's needs.” (N22, 31 years old, F).

\section{c) Increasing cultural awareness and not feeling ignored}

Early recognition of cultural differences was recognized as important by many nurses. They indicated the crucial impact of awareness of cultural issues which can support the survivors' recovery process. One nurse stated:

"From my perspective, the main point is to be aware of the patient's cultural uniqueness as this is something they need... and is helpful in their recovery." (N6, 31 years old, M).

However, despite these recognition survivors indicated these important aspects of themselves were often overlooked or ignored. They wish to feel understood by nurses: "Sometimes nurses seem unaware of our cultural needs... I know they have a busy schedule, but often our needs have a very close relationship with our health... but I have cancer and I want to be alone..." (S8, 43 years old, $M)$. 
"Sometimes I feel invisible.... This makes me feel uncomfortable. I wish nurses were more aware... I wonder if this wish is selfishness?... but I only want to feel better...”

\section{Cross-cultural Care Expectations}

Cultural issues include values, and beliefs that impact healthcare practices, needs, healthseeking behaviors, and physical variations. These beliefs influence health practices which are essential in handling an illness. Culturally based perceptions of cancer survivors can lead to different expectations about the role of health services in responding to their needs. Thus nurses should be sensitive and respectful in assessing, evaluating and adjusting care plans to meet these unique culturally defined needs. Otherwise, nursing care and treatment may be compromised and survivor's health-seeking behaviors and collaboration with nurses can be weakened, leading to a failure to deliver culturally sensitive care.

\section{a) Sensitiveness toward cultural beliefs}

Culturally sensitive care was described as respectful, responsive, resource-connecting, and focused on establishing a therapeutic relationship between survivors and nurses. It adapts to individual survivor needs, beliefs, and behaviors. Therefore, it is essential in providing better support and to be inclusive of differences. By being sensitive to differences, nurses can recognize beliefs and their impacts on the survivors' care perspectives and health-seeking behaviors. One nurse stated:

"If I noticed any cultural needs of the patient, I am acting according to the patient's expectations. But, it is not easy... learning and then practicing ways of providing culturally sensitive care for all include both awareness and sensitivity to others." Another nurse reported: "Some patients are like closed box, you never know of his feelings, thoughts, beliefs... etc. But if I noticed I can help to him...” (S1, 57 years old, F).

Additionally, developing an understanding of our own cultural background and the ways that others' cultural backgrounds influence personal attitudes, values, and beliefs is also 
an important step to using culturally appropriate interventions and being sensitive to others. In this regards, one survivor highlighted an apparent absence of empathy from the nurse:

"I was asking about everything even about the feet. For example, I was asking if I could eat ..., but the nurse warned me not to ask so many questions. Yes, it can be simple for her, but it was difficult for me... It was about my culture.” (S8, 43 years old, $M)$.

Similarly, a nurse emphasized how empathy helped her in being culturally sensitive. “My mum was a cancer patient, and, I cannot even imagine ignoring patients' needs particularly personal and cultural. When the patient tries to stand on his feet, we should help him fully. So, yes I am sensitive to cultural needs.” (N6, 31 years old, M).

Another nurse reported:

"I am as a nurse doing what is necessary. My care is adapted to cultural differences. For example, if a patient wants to listen to his favorite folk music or pray at home, he can do it. It helps him feel relaxed and improve wellbeing, I encourage this kind of cultural activity as my aim is to support the patient, not limit..." (N12, 28 years old, F).

\section{b) Respect toward different cultures}

Engaging culturally with people involves a typically humanistic interpersonal stance, which will attenuate the tendency for nurses to overvalue their own perspectives and worldviews, but instead join with the survivor to explore their perspective and worldview. Having respect for cultural norms has greater value in understanding the meaning of culture and its importance in healthcare delivery. Survivors indicated the value of respect during their traumatic experiences:

“Nurses have to balance their relations. Where my freedom begins, the nurses' finishes." Another was critical of his experience: 
"There is no respect in the health care system. I respect people's beliefs, values, etc. and, I expect them to respect mine too. Especially in the hospital, you are needing respect...” (S14, 49 years old, $F)$.

Whilst nurses recognized the importance of flexibility toward cultural differences, they saw the value of respect as a first step:

"I think, respect and being flexible is an important part of cultural care. If everyone except differences and being respectful, there will be better communication between patient and nurse and there will be no problem arising from cultural differences...” (N18, 28 years old, $M)$.

However, some felt that such respect needed to be mutual otherwise this created tensions in the relationship:

“I can respect patients' culture, however, they need to respect me, too. It includes mutual action. If a patient does not respect me, I struggle....” (N22, 29 years old, M).

This struggle was a common experience due to the volume and range of cultural diversity:

"People with cancer travel through a very difficult journey. As a nurse I should notice the cultural values of patients and respect them, ...at least I can do this but often, it can be impossible...” (N27, 25 years old, F).

Another said: "I am human and I can make mistakes, I cannot be sure what all my patient wants, thinks or believes, but I can only do my best to observe and respect his choices and wishes...” (N14, 39 years old, $F)$.

This clash between the ideal and the reality is explored further in the next theme.

\section{Reality in Cross-cultural Care Activities}

Nurses and survivors described barriers to optimum care rooted in cultural values. This typically occurred when a survivor had difficulty reconciling his/her needs, language differences, work overload of nurses, and lack of awareness amongst nurses and survivors. 


\section{a) Language differences as a barrier}

Language was essential in achieving quality care for survivors through being able to communicate their needs or express deeper feelings to nurses and to understand healthcare advice. However, language differences between nurses and survivors can lead to some problems in health care delivery. This barrier was cited by survivors as a cause of neglect:

“...When they know you don't know the language, some of the hospital staff, such as nurses, don't care for you like the others. You feel alone with your cancer" (S18, 50 years old, F).

One nurse described such an example:

"A patient was a lecturer at a university and he did not speak Turkish. We tried to communicate... I tried but did not know very good English. While talking I talked loudly and I guess he thought I was shouting at him. It was difficult to communicate effectively.” (S23, 57 years old, F).

When survivors speak a different language than the nurse, which is an increasing issue in Turkey, it is difficult to determine the survivor's understanding of health experiences or their perceptions of health care. When language barriers can be overcome, trust and good communication improves. One indicated he felt safer when he can express himself in his mother language:

"Trust is everything in the illness process. I want to trust my nurse to be able to talk in my own language about my needs especially the cultural ones. (S6, 44 years old, M).

Some talked about their efforts in coping with language barriers. One indicated how hard she tries:

"First of all, I try to communicate with patients using body language or speaking English if they know English better than Turkish. What he/she needs in cancer process, I can only understand by communicating so if patients have a different culture and language, I make 
much more effort to focus on their needs much more than usual. Because they cannot express themselves fully as a result of the language barrier.” (N13, 33 years old, M).

Another recognized the importance of written and translated information:

"Patients have deeper needs than usual, we notice. We can face difficulties in communicating, but patients can get tailored information from health care providers, usually from nurses, and we have to be sensitive to their needs." (N15, 32 years old, F).

One further way to overcome language barriers was to get help from translators. However, this was not without difficulty as one survivor describes:

'The translator sometimes feels like... .an exercise in futility, you have to make people understand what you need.' (S20, 31 years old, F).

However, a nurse indicated that the translator could be the difference between life and death:

"First I try hard to get information about the culture and language of the patient and then I call a translator. Sometimes the translator can be really helpful. But this needs to be used carefully as you know, culture and language are difficult issues and in translating the real meaning can be lost. However, if there is no other option, the translator can be seen as an essential life preserver" (N20, 31 years old, F).

\section{b) Overload of nurses as a barrier}

Work overload was frequently raised as a factor preventing nurses from providing culturally sensitive care. They have to address many things during a shift, which limits time available to understand the deeper needs of survivors. One survivor stated:

“I don't think cross-cultural care can be integrated into standard oncology care unless heavy workloads of nurses' decrease. Hence, I do not expect to get my cultural needs met.” (S4, 48 years old, F). 
Similarly, one nurse stated her busy schedule in oncology and the ongoing risk of depleted individual resources was also associated with satisfaction in the role:

"Usually I have to care for too many patients than I can handle. Hourly vital signs, monitoring. giving chemotherapy etc. I don't think I will sincerely focus on cultural needs. If I could have fewer patients and more time, I would really like to be sensitive to the culture which may also help me feel more competent in my work.” (N12, 29 years old, F).

Another said:

"I often have too much work to do, and often in a hurry, I cannot finalize the routine care. Hence, it seems an impossible task to fully understand the cultural needs of patients” (N22, 31 years old, $F$ ).

Survivors said their expectations are lowered to only expect basic routine care. Hence, there was limited opportunity to even share their culture-related needs. One indicated: " $M y$ experience...is not a good one, because nurses always have so many things to do and, even if I really have specific cultural needs, I cannot share these with a nurse during her work. I once I tried, but she could not finish her other work and find the time and so I just felt guilty." (S27, 38 years old, $F)$.

\section{c) Lack of awareness toward different cultures}

Lack of knowledge and understanding of cultural needs can be a major limitation to helpseeking. The lack of cultural recognition and open discussion may lead nurses to dismiss or deny survivors' needs and increase poor communication and collaboration. Even those survivors who are able to identify their needs and who want help may be unwilling to seek it. One emphasized that he preferred not to seek help:

"I am from a little town. Sometimes we (I and the nurse) do not understand each other even when using the same language. It looks weird and I lose my confidence and willingness to 
share... I prefer not to express my needs, I think they do not even realize me and my needs....” (S29, 60 years old, $M)$.

This revealing statement highlights what some nurses prioritize:

"There are many patients and the priority is the chemotherapy, culture is for later. Hence I think no one can fully focus on cross-cultural care as to do so is a very optimistic perspective and I am not sure of the real benefit or meaning of culture-specific care” (N18, 25 years old, F).

Such attitudes to culturally sensitive care was not untypical as reflected in the following two comments:

"I think it is very difficult to assess how somebody would need help according to culture. I have got to learn about culture of patients, but it means is extra work and time. This is up to the nurse's willingness, if I want to learn about culture, yes I can do that... but if I do not want, then I do not have to think about cultural needs...” (N23, 30 years old, F).

"I do not think I can notice or do something different for the cultural needs of patients. It is not necessary. This is not insensitivity of course... but culture is not a big issue for patients... I do not think they can feel better or comfortable with cultural support. They only feel relaxed through better communication.” (N12, 29 years old, F).

However, some considered this was down to training and competence:

"Many times it is hard to notice cultural needs, it made me feel blind... This shows I am not competent enough to deliver cultural care. Sometimes I think if we (nurses) chose not to notice those needs deeply... this is easier....” (N20, 31 years old, F).

\section{DISCUSSION}

Cultural competence is critical to providing relevant, effective, and culturally responsive healthcare. Meeting cultural needs strongly impacts on quality of life and becomes increasingly important as the person progresses through the care journey (Patton, 2015; 
Saxena, 2006). In oncology, it requires nurses to be able to identify and respond to cultural needs in order to positively impact on the cancer survivor's journey to recovery.

To our knowledge, this study is the first to explore cancer survivors and oncology nurses' perceptions of cultural care received during the cancer treatment process in Turkey and reveals significant limitations in the delivery of cross-cultural care. Although Turkey is a multicultural and multilingual society, the health care system is largely geared toward serving Turkish-speakers. Thus survivors with little or no proficiency in Turkish are at risk of experiencing language as a health care and cultural barrier which leads to reduced survivor satisfaction, poor cultural care and reduced access to services. This, as Timmins (Donovan et al, 2011) points out, raises the risk of poorer health outcomes. Many participants indicated they found it difficult to care for survivors from other cultures due to these barriers. An overriding issue in this and other studies was lack of time to deliver culturally competent care due to lack of time and work overload. It is difficult to determine whether this was purely a rationalization, compensating for lack of confidence, or culturally barrier. However, work overload is a prevalent stressor for many healthcare professionals and we know that overwork can be associated with depression, and emotional exhaustion amongst nurses which can limit communication and impact the quality of care (Weigl et al., 2016). Interestingly, work overload and "being too busy" was witnessed by many survivors which provoked feelings of guilt when they sought to express their needs to already busy staff. This is an issue that service managers and budget holders need to carefully consider, not only for the quality of person-centered care but also for the well-being of their own staff. Language competence is therefore essential to support effective cross-cultural communication and this can be greatly enhanced through the effective use of translators to bridge the divide between nurse and survivor. 
The results suggest that nurses have difficulties in noticing the unique belief systems of cancer survivors, which can result in insensitivity. Cultural sensitivity, including overcoming barriers, in the context of the acknowledged causes and progression of cancer, can impact health-seeking behaviors (Licqurish et al., 2014). As Schrank et al. (2017) points out it is a core component of person-centered cancer care that requires culturally-competent approaches in order to ensure survivors' well-being. Attending cultural sensitivity helps develop professionally appropriate attitudes, values, and beliefs and enhances the active participation of survivors in their care, including the need for unconditional care (Tee, 2016). Worryingly, some responses suggested that respecting cultural differences was conditional on the nurse themselves receiving respect, raising questions about prevailing attitudes that could inhibit cultural engagement. Perhaps training in both the intrapersonal and interpersonal components which addresses the concept of humility may help here (Davis et al., 2011).

It was also apparent that nurses lack awareness of cross-cultural care in an oncology setting. Without a critical awareness of cultural grounding, people risk imposing their assumptions, and preconceived notions, practices, and values on cancer survivors in their care. Training in this area requires consideration of the moral principles and ideas that underlie clinical humanism through which nurses can develop an awareness of cultural issues in terms of understanding and respect for cancer survivors, from different cultural backgrounds (Tee, 2016). Good communication is fundamental to the delivery of personcentered care, yet cultural differences between nurses and survivors, which trigger prejudice, can influence survivor-nurse communication, potentially leading to negative outcomes (Christopher et al., 2014). Communication is, as Gopal explains, the process by which individuals interact and influence each other through shared cultural patterns and social structures (Gopal, 2011). As multiculturalism and greater ethnicity increases, this study reveals that nurses in Turkey need to learn to raise their awareness, understanding, and 
tolerance, in order to provide appropriately tailored care and to avoid professional challenge and complaint.

Survivors pointed out the importance of feeling valued as humans. The specific expectations from cancer survivors were to receive holistic care, during their cancer journey. This is crucial given the nature of cancer treatment and the potential for end of life decisions. Survivors were very critical that their expectations were often not met and cultural barriers prevented them from receiving, what in their eyes was optimal care. Consequently, several preferred not to share their needs or communicate. What seems evident is the need for oncology nurses to have the interpersonal skills to develop relationships of openness and trust that will facilitate cultural awareness, exchange, and engagement.

Cultural competence consists of developing awareness and sensitivity to cross-cultural differences and similarities, in particular, the specific challenges cancer survivors face in managing and adapting to their illness. Renzaho et al. (2013) reviewed all studies of the effectiveness of cultural competence programs between 2000 and 2011 and found that training in cultural competence helped practitioners enhance their cultural sensitivity. Effective training creates an environment that promotes better emotional catharsis, and enhanced cooperation with survivors. Survivors commonly reported that nurses lack a deep understanding of the cultural aspects of their cancer journey. Oncology nurses have the opportunity to communicate with survivors to sensitively explore a cancer diagnosis, educate regarding expected trajectory, and provide empathy and psychological support. These psychosocial elements of oncology care may not receive sufficient attention when nurses prioritize the physical aspects of care i.e. medical treatment, chemotherapy, etc. This may be compounded for people with different cultural backgrounds resulting in their needs being ignored during routine cancer care (Jeffreys, 2015). Perhaps more concerning was the 
apparent indifference amongst some nurse participants to cultural needs, suggesting the need for some targeted staff development.

According to Leininger's theory, cognition-based nursing care is important in helping, supporting, facilitating and empowering cancer survivors. Additionally, it reveals the different dimensions of culture, including values, health beliefs, religion, and philosophy as important elements of cultural competency (Leininger, 2002). Nurses need to understand and observe the behaviours of cancer survivors, in order to integrate their knowledge of cultural values and beliefs, with treatment effectiveness (Campinha-Bacote, 2002). Ultimately, this study shows that effective nursing is about developing a care philosophy which will enhance survivors feelings of cultural safety which in turn can strengthen their relationships with nurses and improve treatment outcomes.

\section{Strengths and Limitations}

The study is limted by the socio and cultural diversity of participants. Despite this potential limitation, we achieved saturation of data and provided a detailed picture of the perspectives of cancer survivors and nurses on cultural needs in cancer care. Moreover, our study is, to our knowledge, the first to investigate these perspectives in Turkey.

\section{Implications for Practice}

The journey for cancer survivors can be painful and distressing with health care professionals having the essential role of identifying, specifying and meeting cultural care needs. Nurses, as 24-h care providers, are important sources of psychosocial care, and thus their awareness and competence are critical for achieving positive outcomes. Cultural competency needs to be increased with awareness, sensitivity, knowledge, and acceptance of cultural differences.

Raising such awareness is best achieved through the involvement of survivors in the training and development of staff. Firstly, we suggest that oncology nurses need to recognize 
that being sensitive to cultural differences and willing to help cancer survivors can build effective and humanistic approaches that can prevent discrimination in oncology care and positively impact cancer survivor outcomes. Second, nurse educators and managers can work together to develop continuing education and training programs to improve nurses' crosscultural competency, particularly in relation to the goals and processes of oncology care. Lastly, we suggest that a relatively small investment in competency development in oncology services, holds great promise for improving early awareness and sensitivity towards the cultural needs of a vulnerable group of people.

\section{CONCLUSIONS}

This study sought to contribute to our understanding of the cultural needs of cancer survivors from the perspectives of both survivors and nurses working in oncology services in Turkey. It is evident that delivering culturally competent cancer care is essential to today's oncology practice, requiring nurses to appreciate and adapt their care to the cultural needs of survivors.

The data reveals that the relationship between the nurse and cancer survivor is critical to an awareness of cultural needs but that nurses need help in raising their own awareness and appreciation of the benefits and impact of culturally competent care. This includes developing the attitude and confidence to explore differences through appropriate communication and overcoming barriers arising from language and time. It is also evident that managers and those in decision-making positions need to look carefully at introducing cross-cultural education into oncology services to enable the delivery of culturally competent care, consistent with standards around the world. It is only through creating self-awareness amongst nurses, in the context of each survivor and their needs and beliefs, will the standards of practice improve delivering the health outcomes that those in their care deserve.

\section{REFERENCES}


Brown, O., Ham-Baloyi, W. T., Rooyen, D. R. V., Aldous, C., \& Marais, L. C. (2016). Culturally competent patient-provider communication in the management of cancer: An integrative literature review. Global health action, 9(1), 33208.

Campinha-Bacote, J. (2002). The process of cultural competence in the delivery of healthcare services: A model of care. Journal of transcultural nursing. 13(3), 181-184.

Christopher, J.C., Wendt, D.C., Marecek, J., et al. 2014. Critical cultural awareness: Contributions to a globalizing psychology. American Psychologist. 69(7), 645.

Consolidated criteria for reporting qualitative research (COREQ): a 32-item checklist for interviews and focus groups. http:/www.equator-network.org/reporting-guidelines/coreq/. Accessed March 21, 2019.

Cordova, M.J., Riba, M.B., Spiegel, D. 2017. Post-traumatic stress disorder and cancer. Lancet Psychiatry. 4(4), 330-338.

Davis, D. E., Hook, J. N., Worthington Jr, E. L., et al. 2011. Relational humility: conceptualizing and measuring humility as a personality judgment. Journal of Personality Assessment. 93, 225-234.

Donovan, R., Williams, A., Stajduhar, K., et al. 2011. The influence of culture on home-based family caregiving at end-of-life: a case study of Dutch reformed family care givers in Ontario, Canada. Soc Sci Med. 72(3), 338-346.

Douglas, M.K., Rosenkoetter, M., Pacquiao, D.F., et al. 2014. Guidelines for implementing culturally competent nursing care. Journal of Transcultural Nursing. 25(2), 109-121.

Flick, U. 2018. An introduction to qualitative research. UK: Sage Publications Limited.

Fusch, P.I., Ness, L. R. 2015. Are we there yet? Data saturation in qualitative research. Qual Rep. 20(9), 1408-1416. 
Gopal, A. 2011. Internationalization of higher education: Preparing faculty to teach crossculturally. International Journal of Teaching and Learning in Higher Education, 23(3), 373381.

Huang, Y. L., Yates, P. Prior, D. 2009. Factors influencing oncology nurses' approaches to accommodating cultural needs in palliative care. Journal of Clinical Nursing, 18(24), 34213429.

Jeffreys, M.R. 2015. Teaching cultural competence in nursing and health care: Inquiry, action, and innovation. Springer Publishing Company.

Leininger, M. 2002. Culture care theory: a major contribution to advance transcultural nursing knowledge and practices. J Transcult Nurs. 13(3), 189-192.

Leininger, M. 1991. Transcultural care principles, human rights, and ethical considerations. J Transcult Nurs. 3(1), 21-23.

Leininger, M. 1999. What is transcultural nursing and culturally competent care? . J Transcult Nurs. 10(1), 9.

Licqurish, S., Chiang, P., Walker, J., et al. 2014. Cultural beliefs about cancer influencing helpseeking and symptom appraisal: a meta-synthesis of qualitative findings. Journal of Clinical Oncology. 10 (Supplement S9), 118.

Lu, Q., Wong, C.C.Y., Young, L., et al. 2017. Expressive Writing Among Chinese American Breast Cancer Survivors: A Randomized Controlled Trial. Health Psychology. 36(4), 370379.

Malterud, K., Siersma. V.D., Guassora, A.D. 2015. Sample size in qualitative interview studies guided by information power. Qual. Health Res. 26:1753-1760.

Markova, T., Broome, B. 2007. Effective communication and delivery of culturally competent health care. Urologic Nursing. 27(3), 239. 
Maxwell, J. 2013. Qualitative Research Design: an Interactive Approach. 3 ed. Sage Publications, Thousand Oaks.

Moore, M.L., Moos, M.K., Callister, L.C. 2010. Cultural competence: An essential journal for perinatal nurses. White Plains, NY: March of Dimes Foundation.

Nardi, D., Waite, R., Killian, P. 2012. Establishing standards for culturally competent mental health care. J Psychosoc Nurs Ment Health Serv. 50(7), 3-5.

Neergaard, M.A., Olesen, F., Andersen, R.S., et al. 2009. Qualitative description - the poor cousin of health research? BMC Med. Res. Methodol. 9, 52.

Palmer, N.R., Kent, E.E., Forsythe, L.P., et al. 2014. Racial and ethnic disparities in patientprovider communication, quality-of-care ratings, and patient activation among long-term cancer survivors. J Clin Oncol. 32(36), 4087-4094.

Patton, M.Q. 2015. Qualitative Research \& Evaluation Methods: Integrating Theory and Practice. California: SAGE Publications, Inc., Thousand Oaks.

Rao, A.A.N., Warad, D.M., Weaver, A.L., et al. 2018. Cross-Cultural Medical Care Training and Education: a National Survey of Pediatric Hematology/Oncology Fellows-in-Training and Fellowship Program Directors. Journal of Cancer Education. 1-9.

Renzaho, A.M.N. Romios, P. Crock, C. et al. 2013.The effectiveness of cultural competence programs in ethnic minority patient-centered health care - a systematic review of the literature, Int. J. Qual. Health Care. 25(3), 261-269.

Rosalie, D. T., Sandra, C. T. 2013. Refining the concept of cultural competence: building on decades of progress. Med J Aust, 199(1), 35-8.

Sandelowski M. (2010). What's in a name? Qualitative description revisited. Res. Nurs. Health. 33, 77-84.

Saxena, S. (2006). A cross-cultural study of spirituality, religion, and personal beliefs as components of quality of life. Soc Sci Med. 62(6), 1486-1497. 
Schrank, B. Rumpold, T. Amering, M. et al. 2017. Pushing boundaries-culture-sensitive care in oncology and palliative care: a qualitative study. Psycho-Oncology. 26, 763-769.

Sharifi, N., Adib-Hajbaghery, M., Najafi, M. (2019). Cultural competence in nursing: A concept analysis. International journal of nursing studies. 99, 103386.

Sheldon, L.K., Harris, D., Arcieri, C. (2012). Psychosocial concerns in cancer care: the role of the oncology nurse. Clin. J. Oncol. Nurs. 16(3), 316-319.

Sulstarova, B. (2016). Cross-cultural communication in oncology: challenges and training interests. Oncology nursing forum 43(1), E24.

Surbone, A. (2008). Cultural aspects of communication in cancer care. Support Care Cancer. $16,235-240$.

Tee, S. (2016). Person-centred Approaches in Healthcare. A handbook for Nurses and Midwives, McGraw Hill, London, UK.

The National Coalition for Cancer Survivorship (NCCS). Web site. https://www.canceradvocacy.org. Accessed March 21, 2019.

Vallurupalli, M., Lauderdale, K., Balboni, M., et al. (2012). The role of spirituality and religious coping in the quality of life of patients with advanced cancer receiving palliative radiation therapy. J Support Oncol. 10(2), 81-87.

van Eechoud, I. J., Grypdonck, M., Beeckman, D., Van Lancker, A., Van Hecke, A., \& Verhaeghe, S. (2016). Oncology health workers' views and experiences on caring for ethnic minority patients: a mixed method systematic review. International Journal Of Nursing Studies, 53, 379-398.

Weber, O., Sulstarova, B., Singy, P. 2016. Cross-Cultural Communication in Oncology: Challenges and Training Interests. Oncology Nursing Forum. 43(1), 24-33. 
Weigl, M., Stab, N., Herms, I., et al. 2016. The associations of supervisor support and work overload with burnout and depression: a cross-sectional study in two nursing settings. JAN. 72(8), 1774-88.

You, J., Lu, Q., Zvolensky, M.J., Meng, Z., et al. 2017. Anxiety- and Health-Related Quality of Life Among Patients With Breast Cancer: A Cross-Cultural Comparison of China and the United States. J. Glob. Oncol. 4, 1-9. 